\title{
Autophagy Promotes Peripheral Nerve Regeneration and Motor Recovery Following Sciatic Nerve Crush Injury in Rats
}

\author{
Hai-cheng Huang ${ }^{1} \cdot \mathrm{Li} \mathrm{Chen}^{1} \cdot$ Hai-xing Zhang ${ }^{1} \cdot{\text { Sheng-fa } \mathrm{Li}^{1}}^{1}$ Pei Liu ${ }^{1}$ • \\ Tian-yun Zhao ${ }^{1} \cdot$ Chuan-xiang $\mathrm{Li}^{1}$
}

Received: 2 December 2014 / Accepted: 27 October 2015 /Published online: 7 January 2016

(C) Springer Science+Business Media New York 2016

\begin{abstract}
Autophagy maintains cellular homeostasis by stimulating the lysosomal degradation of cytoplasmic structures, including damaged organelles and dysfunctional proteins. The role of autophagy in the renewal and regeneration of injured peripheral nerves remains poorly understood. The current study investigated the role of autophagy in peripheral nerve regeneration and motor function recovery following sciatic nerve crush injury in rats by stimulating or suppressing autophagy and detecting the presence of autophagosomes and LC3-II expression by electron microscopy and Western blotting, respectively. Neurobehavioral function was tested by CatWalk gait analysis $1,2,3$, and 6 weeks after injury, and the expression of neurofilament (NF)-200 and myelin basic protein (MBP) at the injury site was examined by immunocytochemistry. Apoptosis at the lesion site was determined by the terminal deoxynucleotidyl transferase dUTP nick end labeling assay. Treatment of injured rats with the autophagy inducer rapamycin increased the number of autophagosomes and LC3-II expression while reducing the number of apoptotic cells at the lesion; this was associated with an upregulation of MBP and NF-200 expression and increased motor function recovery as compared to sham-operated rats and those that were subjected to crush injury but untreated. The opposite effects were observed in rats treated with the autophagy inhibitor 3-methyladenine. These data indicate that the modulation of autophagy in peripheral nerve injury could be
\end{abstract}

Hai-cheng Huang and Li Chen have equal contribution.

Chuan-xiang Li

lcxdoc@163.com

1 Department of Anesthesiology, The Third Affiliated Hospital of Southern Medical University, 183 West Guangzhou, Avenue, Guangzhou 510630, China an effective pharmacological approach to promote nerve regeneration and reestablish motor function.

Keywords Peripheral nerve regeneration · MTOR · Autophagy $\cdot$ Rapamycin $\cdot$ Rat

\section{Introduction}

Damage to peripheral nerves leads to Wallerian degeneration and disruption of axonal connections at the lesion site (Sta et al. 2014; Chen et al. 2007; Fahr and Sauser 1988; Koltzenburg 2004), while a strong inflammatory response, oxidative stress, and nutritional deficiency further exacerbate the negative impact at early stages. Previous studies have shown that Schwann cells (SCs) occupy the most part of the peripheral nervous system, accounting for 70 80 \% (Madduri and Gander 2010). Also, Schwann cells (SCs) play a key role in nerve regeneration and the recovery of motor function (Madduri and Gander 2010; Raivich and Makwana 2007), by secreting a variety of neurotrophic factors such as neurotrophin-3, soluble protein-100, myelin basic protein (MBP) (Forghani et al. 2001), and neurofilament (NF)-200 (Sotelo-Silveira et al. 2000) -all of which promote myelination and axonal growth - and also by phagocytosis of damaged axons and myelin, thereby restoring a favorable growth environment (Fu and Gordon 1997).

Autophagy is a conserved intracellular mechanism for maintaining cellular homeostasis in which damaged or dysfunctional proteins, lipids, and organelles are degraded by the lysosome (Levine and Klionsky 2004). Digested components are recycled and provide energy for cell repair (Mizushima and Komatsu 2011). Autophagy is associated with both normal and disease states, such as starvation, neurodegeneration, infection, and aging (Mizushima et al. 2008; Choi et al. 2013). 
Under conditions of stress, autophagy enhances cellular adaptation to prolong survival (Bergmann 2007).

In the early stages of peripheral nerve injury (PNI), damaged or dysfunctional proteins, lipids, and organelles accumulate at the lesion site, creating local stress that hinders the ability of SCs to stimulate nerve repair (Pereira et al. 2012). Autophagic clearance mechanisms can improve the microenvironment and provide basal energy for SC survival. Various studies have demonstrated the protective role of autophagy in the central nervous system with respect to cerebral trauma (Clark et al. 2008), hypoxia-ischemia brain injury (Carloni et al. 2008; Carloni et al. 2010; Jiang et al. 2010; Balduini et al. 2012; Carloni et al. 2014), and acute spinal cord injury (Wang et al. 2014; Hou et al. 2014). Autophagy was found to prevent neurodegeneration in the peripheral nervous system (PNS) in animal models of neuropathy (Kosacka et al. 2013; Qu et al. 2014); in one study, autophagy by SCs blocked the onset and recurrence of neuropathic pain, possibly via a nerve regeneration mechanism (Marinelli et al. 2014). However, the molecular mechanisms of autophagy in the PNS are only partially understood.

This study examined autophagy in the context of peripheral nerve regeneration and its effect on motor function in a rat model of sciatic nerve crush injury (NCI).

\section{Materials and Methods}

\section{Animals and Surgical Procedures}

All protocols were approved by the animal experimental ethics committee of Southern Medical University. Adult female Sprague-Dawley rats weighing 180-220 g were purchased from the Laboratory Animal Centre at the Southern Medical University. Rats were subjected to sciatic NCI as previously described (Raducan et al. 2013). Briefly, rats were deeply anesthetized with sodium pentobarbital $(50 \mathrm{mg} / \mathrm{kg}$ body weight by intraperitoneal injection), and the sciatic nerve in the right mid-thigh was exposed and clamped with a pair of forceps three times for $10 \mathrm{~s}$ each at 10-s intervals. Animals were housed in controlled conditions on a 12:12 h light/dark cycle, with free access to food and water.

\section{Drug Treatment}

Animals were divided into four groups: sham + vehicle (v), crush $+\mathrm{v}$, crush + rapamycin (rapa), and crush $+3-$ methyladenine (3-MA). Rapamycin and 3-MA were obtained from Sigma-Aldrich (St. Louis, MO, USA) and dissolved in $0.5 \%$ dimethyl sulfoxide (DMSO). Animals received daily intraperitoneal injections of rapamycin at a dose of $1 \mathrm{mg} / \mathrm{kg}$ (crush + rapa group), 3-MA at a dose of $50 \mathrm{mg} / \mathrm{kg}$ (crush +3-MA group), or DMSO (crush $+v$ group) for 5 days after the surgery. Sham-operated rats (sham $+v$ group), in which the sciatic nerve was exposed but not subjected to crush injury, received daily intraperitoneal injections of an equal volume $(1 \mathrm{~mL}) 0.5 \%$ DMSO for 5 days. Drug dosages were selected based on data from previous studies and preliminary experiments (Maeda et al. 2013; Chen et al. 2013; Sekiguchi et al. 2012).

\section{Tissue Preparation}

Sciatic nerves were dissected and harvested 1 and 6 weeks post-injury ( $n=6$ for each time point and group). Animals were decapitated and perfused via the left ventricle with cold saline followed by $4 \%$ paraformaldehyde in $0.01 \mathrm{M}$ phosphate-buffered saline (PBS; $\mathrm{pH} 7.35$ ). The injured sciatic nerve was then removed and frozen at $-80{ }^{\circ} \mathrm{C}$ for histological or Western blot analysis.

\section{Immunocytochemistry}

Transverse frozen sections (10- $\mu \mathrm{m}$ thickness) of sciatic nerve were dried, mounted onto slides, and washed in $0.1 \mathrm{M}$ PBS and then blocked in $10 \%$ goat serum and $3 \%$ bovine serum albumin (BSA) for $1 \mathrm{~h}$ at $37^{\circ} \mathrm{C}$. After two washes with PBS, the slides were incubated with primary antibodies against MBP (1:1000; Sigma), NF-200 (1:500; Sigma), or LC3B-II (1: 500; Cell Signaling Technology, Danvers, MA, USA) at $4{ }^{\circ} \mathrm{C}$ for $12 \mathrm{~h}$, followed by repeated washes with PBS, and then incubated with Alexa 488- or Cy3-conjugated secondary antibodies (1:1000; Invitrogen, Shanghai, China) for $2 \mathrm{~h}$ at room temperature. Sections were visualized under an epifluorescence microscope (Leica, DM6000B, Wetzlar, Germany) and imaged at $\times 400$ magnification.

\section{Western blot Analysis}

LC3-II protein expression at the lesion site following sciatic NCI was analyzed by Western blotting. Nerve tissue samples were homogenized in tissue lysis buffer $(20 \mathrm{mM}$ Tris- $\mathrm{HCl}$, pH 7.5; $150 \mathrm{mM} \mathrm{NaCl} ; 1 \%$ Triton X-100; 25 mM NaPPi; $80 \mathrm{mM} \beta$-glycerophosphate; $2 \mathrm{mM}$ EDTA; $0.2 \mathrm{mM} \mathrm{Na}$ VO4 and protease inhibitor cocktail) and centrifuged at $12,000 \mathrm{~g}$ for $15 \mathrm{~min}$ at $4{ }^{\circ} \mathrm{C}$. The protein concentration in the supernatant was measured with a bicinchoninic assay kit (Beyotime Institute of Biotechnology, Shanghai, China), and the supernatant was mixed with sample buffer $(50 \mathrm{mM}$ Tris- $\mathrm{HCl}, 10 \%$ sodium dodecyl sulfate, $10 \%$ glycerol, $10 \%$ 2-mercaptoethanol, and $2 \mathrm{mg} / \mathrm{ml}$ bromophenol blue) at a 1:1 ratio and boiled for $5 \mathrm{~min}$. A total of $20 \mu \mathrm{g}$ of each sample was separated on a $15 \%$ acrylamide gel and transferred to a nitrocellulose membrane (BioScience, Shanghai, China) by electroblotting (BioRad, Hercules, California, USA) at $120 \mathrm{~V}$ for $1.5 \mathrm{~h}$ followed by $70 \mathrm{~V}$ for $0.5 \mathrm{~h}$ at $4{ }^{\circ} \mathrm{C}$. Membranes were blocked with $3 \%$ BSA and incubated with rabbit anti-LC3B-II antibody (1:000) overnight at $4{ }^{\circ} \mathrm{C}$, followed by incubation with an anti-rabbit 
secondary antibody (1:2000; Biosynthesis Biotechnology Co. Ltd., Beijing, China) for $1.5 \mathrm{~h}$ at room temperature. The blots were visualized using Scanmaker 3836 (Microtek Technology Co. Ltd., Shanghai, China) and quantified with Quantity One software (Bio-Rad). The expression level of LC3-II was determined by calculating band intensity relative to $\beta$-actin.

\section{Electron Microscopy}

Sciatic nerve segments were removed 1 week after NCI and fixed with $2.5 \%$ glutaraldehyde overnight at $4{ }^{\circ} \mathrm{C}$. The segments were cut with a sharp razor blade into serial $1-\mathrm{mm}$ transverse sections around the site of injury, which were post-fixed in $1 \%$ osmium tetroxide for $1 \mathrm{~h}$ at $4{ }^{\circ} \mathrm{C}$, rinsed in PBS, dehydrated in a graded series of alcohol and propylene oxide, and embedded in Epon. Blocks showing a predominantly transverse orientation of the injured sciatic nerve were selected from toluidine blue-stained thick sections. Ultrathin $(70-\mathrm{nm})$ sections were prepared on an ultramicrotome (Ultracut R, Leica, Heerbrugg, Switzerland) with a diamond knife, stained with uranyl acetate and lead citrate, and visualized using an a JEM-1010 electron microscope (Jeol, Tokyo, Japan) at $\times 1500$ or $\times 30,000$ magnification.

\section{TUNEL}

DNA fragmentation resulting from cell death was detected in transverse frozen sections $(10 \mu \mathrm{m})$ of sciatic nerves at the lesion site by terminal deoxynucleotidyl transferase dUTP nick end labeling (TUNEL) staining using the TUNEL apoptosis assay kit (Beyotime Institute of Biotechnology) according to the manufacturer's protocol 1 week post-injury. Samples were counterstained with DAPI (blue color illustrates

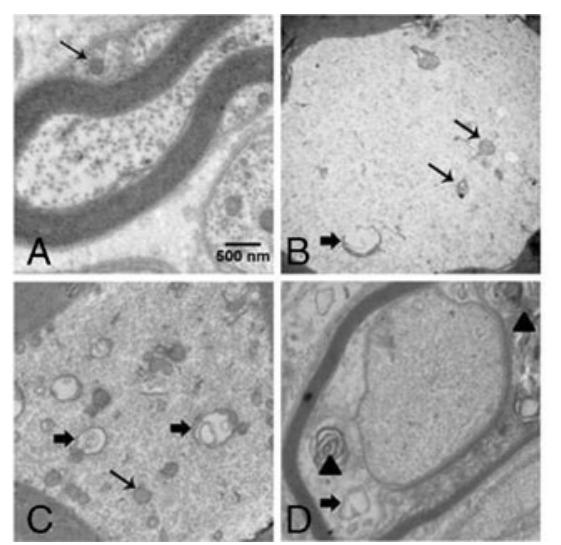

E

Fig. 1 Autophagosomes in the sciatic nerve following PNI. Representative electron micrographs showing autophagosomes and lysosomes (indicated by broad and narrow arrows, respectively) in axons from the sciatic nerve of rats in the following groups: a sham $+\mathrm{v}$ (sham-operated and injected with the vehicle $0.5 \%$ DMSO), $\mathbf{b}$ crush $+\mathrm{v}$ (subjected to NCI and injected with vehicle), $\mathbf{c}$ crush + rapa (subjected to $\mathrm{NCI}$ and injected with $1 \mathrm{mg} / \mathrm{kg}$ rapamycin), and $\mathbf{d}$ crush $+3-\mathrm{MA}$ cellular nuclei) and imaged at $\times 400$ magnification, and the number of TUNEL-positive cells in each section was counted.

\section{Behavioral Analysis}

Experimenters performing the behavioral tests were blinded to the drug treatment conditions. Motor function was determined by stand time and footprint intensity using the CatWalk system (Noldus Inc., Wageningen, Netherlands). Animals crossed a walkway with an illuminated glass floor, and a GP-3360 high-speed video camera (Gevicam, Milpitas, CA, USA) equipped with an 8.5 -mm wide-angle lens (Fujicon Corp., Shenzhen, China) positioned underneath the walkway automatically recorded paw prints as the animals moved across. The test was performed 1, 2, 3, and 6 weeks after injury.

\section{Statistical Analysis}

Data is presented as the mean \pm SEM. Comparisons within groups were made by one-way analysis of variance (ANOVA), and between-groups comparisons were made by one-way ANOVA and, if significant, were followed by discrete comparisons using a post hoc test with a Bonferroni correction for multiple comparisons. $P<0.05$ was considered statistically significant. All analyses were performed using GraphPad Prism 5 software (GraphPad Software, Inc., San Diego, CA, USA).

\section{Results}

Autophagy is enhanced by rapamycin and suppressed by 3MA at the acute stage of PNI. Autophagosomes were visualized by electron microscopy 1 week after sciatic NCI. A 
greater number of autophagosomes were present in the rapamycin-treated (Fig. 1c) and crush $+v$ groups (Fig. 1b); the numbers were eightfold and threefold higher, respectively, than in the sham (Fig. 1a) group and were also higher than in the 3-MA-treated group (Fig. 1d). These data are correlated with an increase of autophagy after NCI. Western blot (Fig. 2b) and immunocytochemical (Fig. 2a) analyses of the expression of the autophagy marker LC3-II revealed higher protein levels in rapamycin-treated animals, and lower levels in the 3-MA group $(P<0.05)$ compared to controls. These results indicate that autophagy is enhanced by rapamycin and suppressed by 3-MA at the acute stage after sciatic NCI.

\section{Autophagy Promotes Motor Function Recovery Following PNI}

The recovery of motor function was assessed using the CatWalk system. The stand time and footprint intensity of the operated limbs were applied to analyze motor function 1, 2, 3 , and 6 weeks post-injury (Fig. 3c, d). The mean stand time in the rapamycin-treated group was twofold higher than in the crush $+\mathrm{v}$ group at 1 and 2 weeks post-injury $(P<0.05)$, while the mean footprint intensity was comparable between the two groups at 2 weeks. These results indicate that animals treated with rapamycin were better able to support their body weight on injured limbs starting 2 weeks after injury, possibly as a result of the enhanced autophagy at the acute stage. In contrast, in the 3-MA group, the mean stand time was significantly lower at 1 and 2 weeks post-injury (45 and $15 \%$, respectively), while the mean footprint intensity was about $20-30 \%$ lower at 1,2 , and 3 weeks post-injury as compared to shamoperated animals $(P<0.05)$.

\section{Autophagy Promotes Axonal Regeneration and SC Remyelination}

MBP is a major constituent of the myelin sheath produced by $\mathrm{SCs}$ in the peripheral nervous system, while the expression of NF-200 - an axon-specific intermediate filament - is critical for axon stabilization during their maturation. To investigate the effects of autophagy on nerve regeneration, the expression of MBP and NF-200 was evaluated by immunocytochemistry.
Fig. 2 Expression of the autophagy marker LC3-II following PNI and drug treatment. The experimental groups are as defined in Fig. 1 caption. LC3-II expression was detected by a immunocytochemistry (red; nuclei are stained with DAPI and appear as blue) and b Western blotting. $\mathbf{c}$ The level of autophagy was determined by calculating the relative ratio of LC3-II to -I expression. Crush $+\mathrm{v}$ group, crush + rapa group, and crush +3 MA group were compared with sham $+\mathrm{v}$ group, respectively. $* * P<0.01, * * * P<0.001, n s$ means no significance (betweengroups comparison with Bonferroni correction; $n=6$ rats/group). Scale bar $=10 \mu \mathrm{m}$

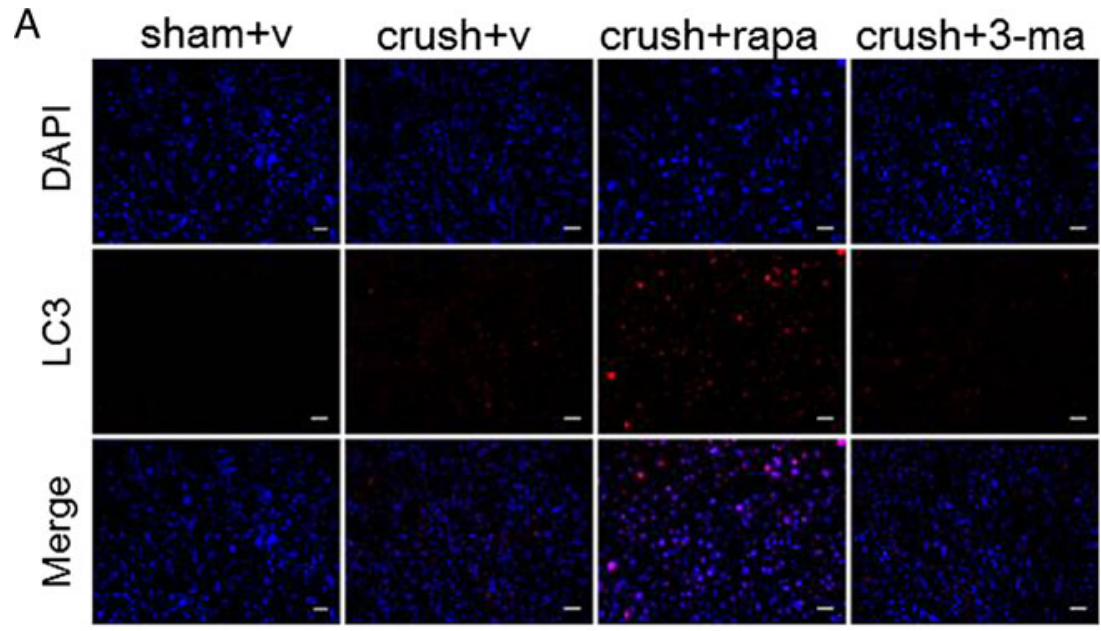

B

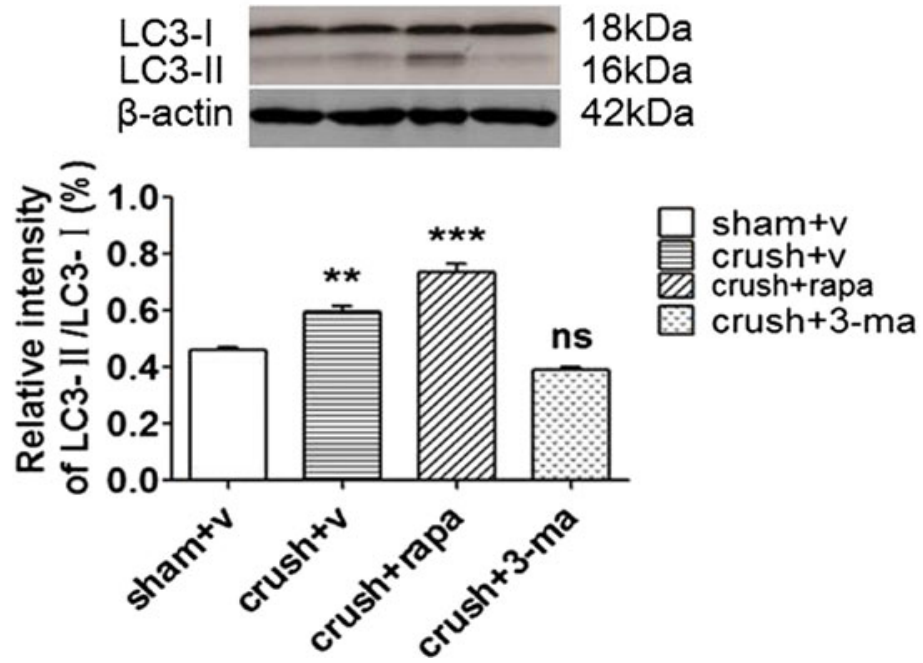




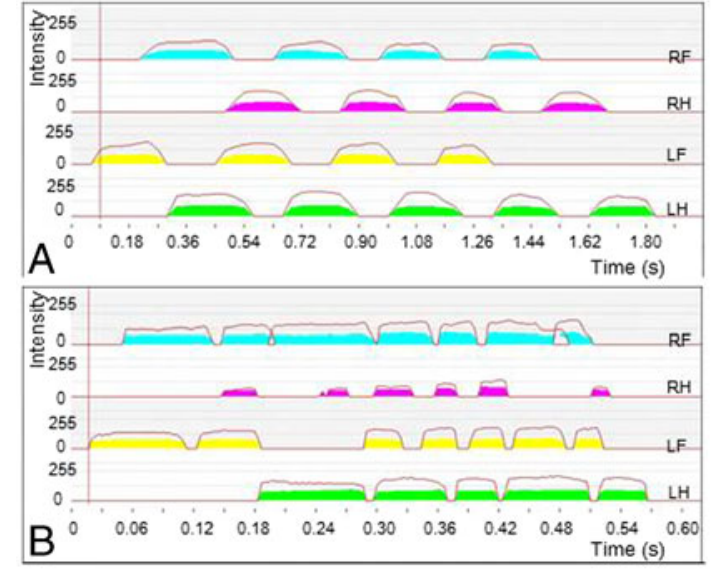

Fig. 3 Recovery of motor function following PNI. Representative CatWalk analysis trace from a a sham-operated rat $(\operatorname{sham}+v)$ and $\mathbf{b}$ a post-injury rat. Groups are as defined in Fig. 1 caption. $L F$, left forelimb; $L H$, left hind limb; $R F$, right forelimb; $R H$, right hind limb. $\mathbf{c}$ Footprint

At 1 week post-injury, at the peak of nerve regeneration, MBP and NF-200 immunoreactivity was 1.4- and 2-fold higher, respectively, in rats treated with rapamycin as compared to those treated with 3-MA or were subjected to injury but untreated $(P<0.001$; Fig. 4). In addition, MBP and NF-200 expression was lower in the 3-MA-treated group relative to the crush $+\mathrm{v}$ group $(P>0.05)$.

Fig. $4 \mathrm{MBP}$ and NF-200 expression following NCI. Protein expression of MBP (green) and NF-200 (red) at the site of injury was evaluated by immunocytochemistry in each experimental group (defined in the Fig. 1 caption). Fluorescence intensity was calculated by the integrated density of pixels for $\mathbf{b}$ MBP and $\mathbf{c}$ NF-200. Crush $+\mathrm{v}$ group, crush + rapa group, and crush + 3-MA group were compared with sham $+\mathrm{v}$ group, respectively. $* P<0.05$, $* * P<0.01, n s$ means no significance (between-groups comparison of integrated fluorescence intensity with Bonferroni correction; $n=6$ rats/group). Scale bar $=20 \mu \mathrm{m}$
A
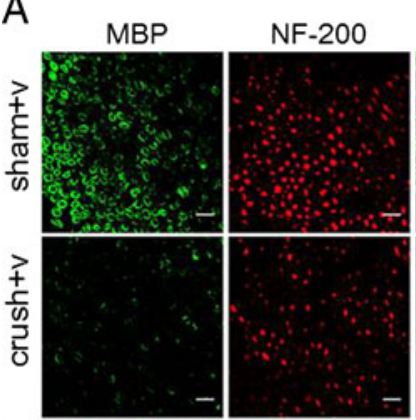

Merge

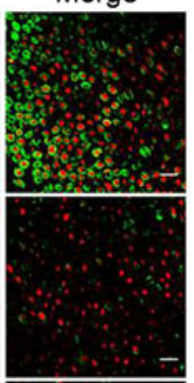

$\frac{\pi}{2}$
$\frac{2}{\pi}$
$\frac{c}{4}$
$\frac{c}{0}$
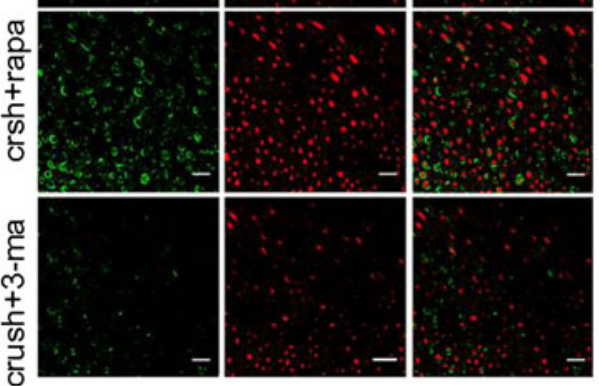

Mean intensity of RH

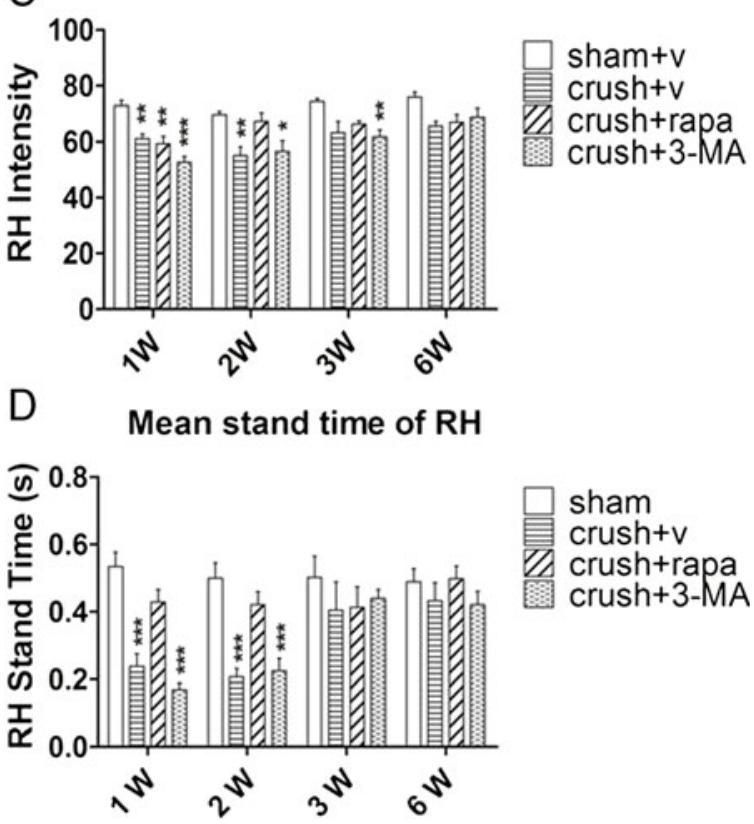

intensity and $\mathbf{d}$ stand time were evaluated 1,2,3, and 6 weeks after injury $\left(1 \mathrm{~W}, 2 \mathrm{~W}, 3 \mathrm{~W}\right.$, and $6 \mathrm{~W}$, respectively). ${ }^{*} P<0.05, * * P<0.01$, $* * * P<0.001$ (between-groups comparison at different time points with Bonferroni correction; $n=6$ rats/group)

The lesion site of the sciatic nerve was examined 6 weeks post-injury by electron microscopy (Fig. 5). Axon number and G-ratio - which is the ratio of inner to outer diameter of the myelin sheath - differed in the four groups (Fig. 4b). There was a greater number of smaller axons (diameter $\leq 4 \mu \mathrm{m}$ ) while larger axons (diameter $>4 \mu \mathrm{m}$ ) were more abundant in the rapamycin-treated and sham-operated groups than in the 
Fig. 5 Axon regeneration and remyelination following PNI. Representative electron micrographs of sciatic nerve axons in a sham $+v$, b crush $+v$, c crush + rapa, and $\mathbf{d}$ crush +3-MA groups (defined in the Fig. 1 caption). e, $f$ The $\mathrm{G}$ ratio was calculated (e) and number of axons was quantified (f) for each group. g Comparisons of $\mathrm{G}$ ratio are shown; downward arrow, lower or decreased expression; upward arrow, higher or increased expression; and dash, no significant difference. $* P<0.05, * * P<0.01$, $* * * P<0.001$ (between-groups comparison of relative abundance of different axon sizes with Bonferroni correction; $n=3$ rats/ group). Scale bars $=10 \mu \mathrm{m}$
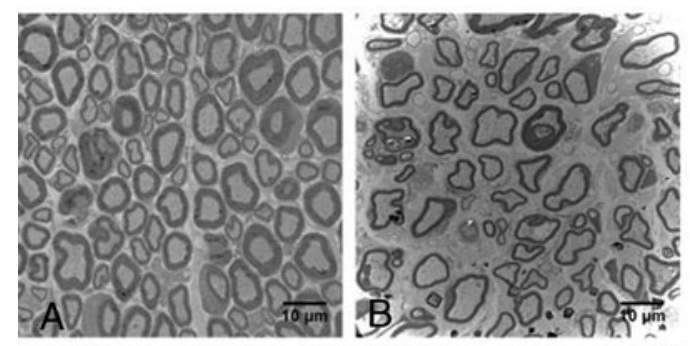

$E$
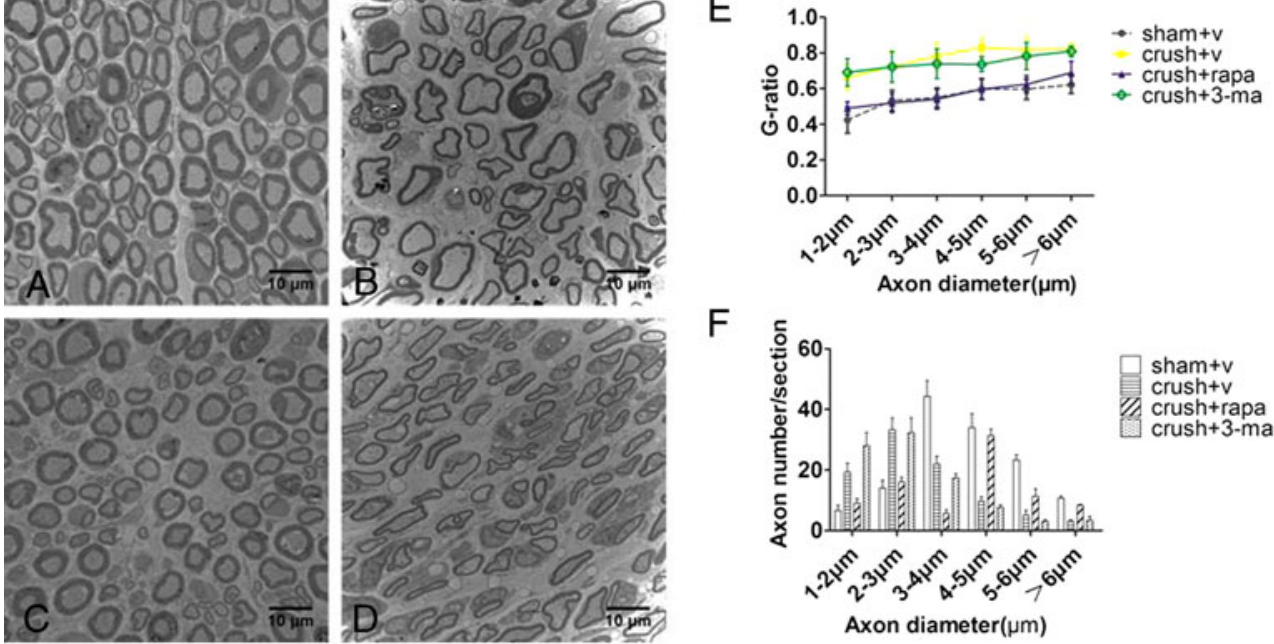

F

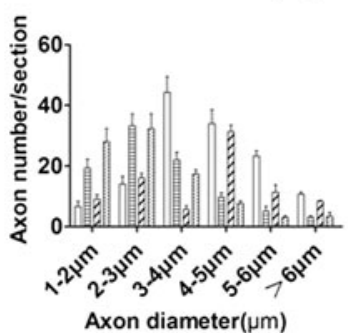

sham $+v$ crush+v

crush+rapa crush+3-ma crush $+\mathrm{v}$ and 3-MA groups, indicating that axon remyelination was delayed in the former two groups. The $\mathrm{G}$ ratio of large axons was $0.59-0.68$ in the crush $+v$ and 3-MA groups, suggesting that axonal maturation was inhibited, whereas in the rapamycin-treated and sham-operated groups, the $G$ ratio was $0.73-0.83$, indicating that axonal maturation was enhanced by activating and suppressed by inhibiting autophagy. Thus, the activation of autophagy improves the remyelination function of SCs as well as axon regeneration.

\section{Autophagy Decreases Cell Death Following Sciatic PNI}

The cytoprotective role of autophagy was investigated by TUNEL staining (Fig. 6). A marked decrease of apoptotic cells was detected in rapamycin-treated animals 1 week post-injury as compared to the crush $+\mathrm{v}$ and 3-MA groups, indicating that the activation of autophagy reduced the number of apoptotic cells following sciatic NCI.
Fig. 6 Autophagy decreases cell death following sciatic PNI. a Cell death in SCs was detected in each experimental group (defined in the Fig. 1 caption) by DAPI (blue) and TUNEL (green) double staining. b Quantitative analysis of TUNEL-positive cells. $* P<0.05$, $* * * P<0.001$ (between-groups comparison of TUNEL-positive cells with Bonferroni correction; $n=6$ rats/group). Scale bar $=10 \mu \mathrm{m}$
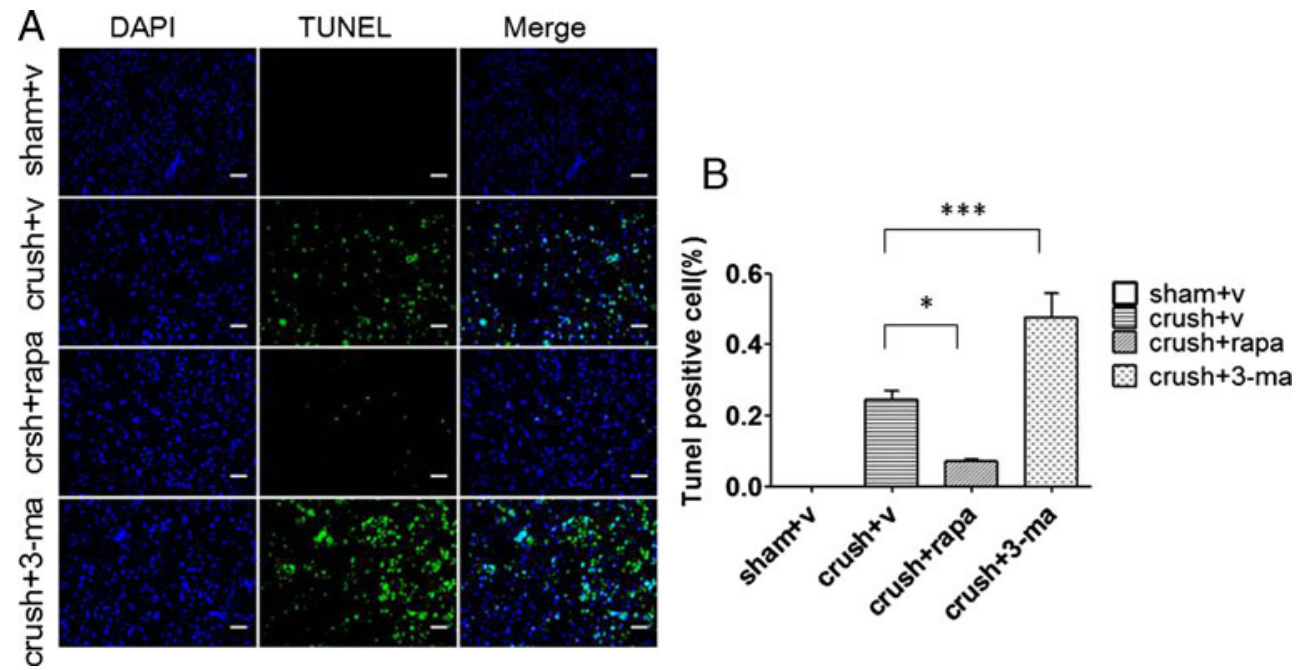


\section{Discussion}

PNI is a common type of trauma that involves Wallerian degeneration and disruption of axonal connections at the lesions site (Sta et al. 2014; Pereira et al. 2012). The mammalian target of rapamycin (mTOR) signaling pathway plays an important role in mediating the regenerative response and functional recovery following injury by regulating cell metabolism, proliferation, death, and survival as well as autophagy (Laplante \& Sabatini 2009), and has also been linked to traumatic brain injury (Clark et al. 2008), neonatal hypoxia-ischemia-induced brain injury (Carloni et al. 2008), and spinal cord injury (Wang et al. 2014). Recent studies have reported that inhibition of mTOR signaling by rapamycin results in the activation of autophagy, which functions to remove damaged cellular components and maintain cell homeostasis (Pattingre et al. 2008; Dunlop and Tee 2014; Xue et al. 2013).

The current study investigated the role of lysosomal clearance mechanisms of autophagy in the injured rat sciatic nerve. We found that rapamycin treatment induced an upregulation of LC3-II expression following injury, consistent with the activation of autophagy via mTOR inhibition. In addition, cell death at the lesion site was reduced in rapamycin-treated rats, corresponding to a greater degree of neuroregeneration and increased recovery of motor function relative to the vehicle treatment group. The opposite result was observed upon administration of 3-MA. These results demonstrate that negative regulation of mTOR signal caused by rapamycin induces autophagy and promotes peripheral nerve regeneration and motor recovery after sciatic NCI.

In the present study, autophagy was demonstrated via detection autophagosomes by electron microscopy, which is considered as the gold standard (Klionsky et al. 2007). In addition, the protein expression of LC3-II was used to assess autophagy (Mizushima 2009). Aside from those treated with 3-MA, all rats showed an increase in autophagy 1 week after NCI. The pathophysiological processes that occur in the injured nerves, including ischemia, inflammation, oxidative stress, and disruption of the blood supply, deplete essential cellular nutrients and are likely responsible for autophagy activation.

SCs provide trophic support for the remyelination of injured axons to reestablish nerve function (Madduri and Gander 2010; Stassart et al. 2013). In the present study, rapamycin-treated rats showed a greater number of SCs and improved remyelination 1 and 6 weeks post-injury as compared to control animals, indicating that mTOR inhibition by rapamycin activated autophagy to promote SC survival and nerve recovery. Autophagy has a protective role after injury under various circumstances with respect to oxidative stress (Moore 2008), inflammatory response (Jan et al. 2012), acute liver injury ( $\mathrm{Lu}$ et al. 2014), and ischemic preconditioning (Yan et al. 2013). The timeline of the improvement in outcome of the rapamycin-treated group suggests that enhanced autophagy at the acute stage after injury helps to reestablish motor function in the long term. In addition, the results demonstrated that inhibiting autophagy delayed remyelination by Schwann cells and the regeneration of injured axons, indicating that a basal level of autophagy is required to maintain nerve homeostasis, which is consistent with the conclusions of other studies (Carloni et al. 2008; Jiang et al. 2010; Sheng et al. 2010). Thus, while studies have variously reported that inhibiting mTOR signaling with rapamycin can have both beneficial and detrimental effects for nervous system recovery (Chen et al. 2013; Rami et al. 2008; Leibinger et al. 2012; Wen et al. 2008; Wang et al. 2012), the results of the current study indicate that activating autophagy in injured peripheral nerves by mTOR inhibition serves a protective function in the early post-injury stages that can ensure the recovery of motor function later on.

In summary, the findings presented here provide evidence supporting a role for autophagy in nerve regeneration and motor function recovery in the PNS and can serve as a basis for the development of therapeutics that improve the outcome of PNI via pharmacological induction of autophagy.

Acknowledgments The authors would like to thank director Chuanxiang Li from the Third Affiliated Hospital of Southern Medical University and Prof. Xiao-chun Bai from School of Basic Medical Science. This work gained equipment supports and technical assistance of teachers from the Department of Cell Biology at Southern Medical University.

\section{References}

Balduini W, Carloni S, Buonocore G (2012) Autophagy in hypoxiaischemia induced brain injury. J Matern Fetal Neonatal Med 25(1): $30-40$

Bergmann A (2007) Autophagy and cell death: no longer at odds. Cell 131(6):1032-1034

Carloni S, Buonocore G, Balduini W (2008) Protective role of autophagy in neonatal hypoxia-ischemia induced brain injury. Neurobiol Dis 32(3):329-339

Carloni S, Girelli S, Scopa C, Buonocore G, Longini M, Balduini W (2010) Activation of autophagy and Akt/CREB signaling play an equivalent role in the neuroprotective effect of rapamycin in neonatal hypoxia-ischemia. Autophagy 6(3):366-377

Carloni S, Albertini MC, Galluzzi L, Buonocore G, Proietti F, Balduini W (2014) Increased autophagy reduces endoplasmic reticulum stress after neonatal hypoxia-ischemia: role of protein synthesis and autophagic pathways. Exp Neurol 255(5):103-112

Chen ZL, Yu WM, Strickland S (2007) Peripheral regeneration. Annu Rev Neurosci 30(1):209-233

Chen HC, Fong TH, Hsu PW, Chiu WT (2013) Multifaceted effects of rapamycin on functional recovery after spinal cord injury in rats through autophagy promotion, anti-inflammation, and neuroprotection. J Surg Res 179(1):203-210

Choi AM, Ryter SW, Levine B (2013) Autophagy in human health and disease. N Engl J Med 368(19):1845-1846

Clark RS, Bayir H, Chu CT, Alber SM, Kochanek PM, Watkins SC (2008) Autophagy is increased in mice after traumatic brain injury 
and is detectable in human brain after trauma and critical illness. Autophagy 4(1):88-90

Dunlop EA, Tee AR (2014) mTOR and autophagy: a dynamic relationship governed by nutrients and energy. Semin Cell Dev Biol 39(14): 121-129

Fahr LM, Sauser DD (1988) Imaging of peripheral nerve lesions. Orthop Clin North Am 19(1):27-41

Forghani R, Garofalo L, Foran DR, Farhadi HF, Lepage P, Hudson TJ, Tretjakoff I, Valera P, Peterson A (2001) A distal upstream enhancer from the myelin basic protein gene regulates expression in myelinforming Schwann cells. J Neurosci. 21(11):3780-3787

Fu SY, Gordon T (1997) The cellular and molecular basis of peripheral nerve regeneration. Mol Neurobiol 14(2):67-116

Hou H, Zhang L, Zhang L, Tang P (2014) Acute spinal cord injury in rats should target activated autophagy. J Neurosurg Spine 20(5):568577

Jan K, Lucia H, Drahomíra H, Irena B, Mouhammed A, Ctirad A (2012) Autophagy and its role in protective and damaging inflammatory response. Cas Lek Cesk 151(3):128-134

Jiang M, Liu K, Luo J, Dong Z (2010) Autophagy is a renoprotective mechanism during in vitro hypoxia and in vivo ischemia-reperfusion injury. Am J Pathol 176(3):1181-1192

Klionsky DJ, Cuervo AM, Seglen PO (2007) Methods for monitoring autophagy from yeast to human. Autophagy 3(3):181-206

Koltzenburg M (2004) Bendszus M (2004) imaging of peripheral nerve lesions. Curr Opin Neurol 17(5):621-626

Kosacka J, Nowicki M, Blüher M, Baum P, Stockinger M, Toyka KV, Klöting I, Stumvoll M, Serke H, Bechmann I, Klöting N (2013) Increased autophagy in peripheral nerves may protect Wistar Ottawa Karlsburg W rats against neuropathy. Exp Neurol 250(10): $125-135$

Laplante M, Sabatini DM (2009) mTOR signaling at a glance. J Cell Sci 122(20):3589-3594

Leibinger M, Andreadaki A, Fischer D (2012) Role of mTOR in neuroprotection and axon regeneration after inflammatory stimulation. Neurobiol Dis 46(2):314-324

Levine B, Klionsky DJ (2004) Development by self-digestion: molecular mechanisms and biological functions of autophagy. Dev Cell 6(4): 463-477

Lu Y, Wang WJ, Song YZ, Liang ZQ (2014) The protective mechanism of schisandrin A in d-galactosamine-induced acute liver injury through activation of autophagy. Pharm Biol 52(10):1-6

Madduri S, Gander B (2010) Schwann cell delivery of neurotrophic factors for peripheral nerve regeneration. J Peripher Nerv Syst 15(2): 93-103

Maeda H, Nagai H, Takemura G, Shintani-Ishida K, Komatsu M, Ogura S, Aki T, Shirai M, Kuwahira I, Yoshida K (2013) Intermittenthypoxia induced autophagy attenuates contractile dysfunction and myocardial injury in rat heart. Biochim Biophys Acta 1832(8): $1159-1166$

Marinelli S, Nazio F, Tinari A, Ciarlo L, D’ Amelio M, Pieroni L, Vacca V, Urbani A, Cecconi F, Malorni W, Pavone F (2014) Schwann cell autophagy counteracts the onset and chronification of neuropathic pain. Pain 155(1): 93-107.

Mizushima N (2009) Methods for monitoring autophagy using GFP-LC3 transgenic mice. Methods Enzymol 452(8):13-23

Mizushima N, Komatsu M (2011) Autophagy: renovation of cells and tissues. Cell 147(4):728-741
Mizushima N, Levine B, Cuervo AM, Klionsky DJ (2008) Autophagy fights disease through cellular self-digestion. Nature 451(7182): $1069-1075$

Moore MN (2008) Autophagy as a second level protective process in conferring resistance to environmentally-induced oxidative stress. Autophagy 4(2):254-256

Pattingre S, Espert L, Biard-Piechaczyk M, Codogno P (2008) Regulation of macroautophagy by mTOR and Beclin 1 complexes. Biochimie 90(2):313-323

Pereira JA, Lebrun-Julien F, Suter U (2012) Molecular mechanisms regulating myelination in the peripheral nervous system. Trends Neurosci 35(2):123-134

Qu L, Liang X, Gu B, Liu W (2014) Quercetin alleviates high glucoseinduced Schwann cell damage by autophagy. Neural Regen Res 9(12):1195-1203

Raducan A, Mirică S, Duicu O, Răducan S, Muntean D, Fira-Mlădinescu O, Lighezan R (2013) Morphological and functional aspects of sciatic nerve regeneration after crush injury. Romanian J Morphol Embryol 54(3):735-739

Raivich G, Makwana M (2007) The making of successful axonal regeneration: genes, molecules and signal transduction pathways. Brain Res Rev 53(2):287-311

Rami A, Langhagen A, Steiger S (2008) Focal cerebral ischemia induces upregulation of Beclin 1 and autophagy-like cell death. Neurobiol Dis 29(1):132-141

Sekiguchi A, Kanno H, Ozawa H, Yamaya S, Itoi E (2012) Rapamycin promotes autophagy and reduces neural tissue damage and locomotor impairment after spinal cord injury in mice. J Neurotrauma 29(5): 946-956

Sheng R, Zhang LS, Han R, Liu XQ, Gao B, Qin ZH (2010) Autophagy activation is associated with neuroprotection in a rat model of focal cerebral ischemic preconditioning. Autophagy 6(4):482-494

Sotelo-Silveira JR, Calliari A, Kun A, Benech JC, Sanguinetti C, Chalar C, Sotelo JR (2000) Neurofilament mRNAs are present and translated in the normal and severed sciatic nerve. J Neurosci Res 62(1): $65-74$

Sta M, Cappaert NL, Ramekers D, Baas F, Wadman WJ (2014) The functional and morphological characteristics of sciatic nerve degeneration and regeneration after crush injury in rats. J Neurosci Methods 222(30):189-198

Stassart RM, Fledrich R, Velanac V, Brinkmann BG, Schwab MH, et al. (2013) A role for Schwann cell-derived neuregulin-1 in remyelination. Nat Neurosci 16(1):48-54

Wang Z, Shi XY, Yin J, Zuo G, Zhang J, Chen G (2012) Role of autophagy in early brain injury after experimental subarachnoid hemorrhage. J Mol Neurosci 46(1):192-202

Wang ZY, Liu WG, Muharram A, Wu ZY, Lin JH (2014) Neuroprotective effects of autophagy induced by rapamycin in rat acute spinal cord injury model. Neuroimmunomodulation 21(5):257-267

Wen YD, Sheng R, Zhang LS, Han R, Zhang X, Zhang XD, Han F, Fukunaga K, Qin ZH (2008) Neuronal injury in rat model of permanent focal cerebral ischemia is associated with activation of autophagic and lysosomal pathways. Autophagy 4(6):762-769

Xue Z, Zhang S, Huang L, He Y, Fang R, Fang Y (2013) Increased expression of Beclin-1-dependent autophagy protects against betaamyloid-induced cell injury in PC12 cells. J Mol Neurosci 51(1): 180-186

Yan WJ, Dong HL, Xiong LZ (2013) The protective roles of autophagy in ischemic preconditioning. Acta Pharmacol Sin 34(5):636-643 